\title{
BMJ Open Informal caregiving for adults, loneliness and social isolation: a study protocol for a systematic review
}

\author{
André Hajek (1) , Benedikt Kretzler, Hans-Helmut König
}

To cite: Hajek A, Kretzler B, König $\mathrm{H}-\mathrm{H}$. Informal caregiving for adults, loneliness and social isolation: a study protocol for a systematic review. BMJ Open 2021;11:e044902. doi:10.1136/ bmjopen-2020-044902

- Prepublication history for this paper is available online. To view these files, please visit the journal online (http://dx.doi org/10.1136/bmjopen-2020044902).

Received 16 September 2020 Revised 19 April 2021 Accepted 20 April 2021
Check for updates

(c) Author(s) (or their employer(s)) 2021. Re-use permitted under CC BY-NC. No commercial re-use. See rights and permissions. Published by BMJ.

Department of Health Economics and Health Services Research, University Medical Center Hamburg-Eppendorf, Hamburg, Germany

Correspondence to

Dr André Hajek; a.hajek@uke.de

\section{ABSTRACT}

Introduction Some empirical studies have identified an association between informal caregiving for adults and Ioneliness or social isolation. However, there is a lack of a review systematically synthesising empirical studies that have examined these associations. Hence, the aim of this systematic review is to provide an overview of evidence from observational studies.

Methods and analysis Three electronic databases (Medline, PsycINFO, CINAHL) will be searched (presumably in May 2021), and reference lists of included studies will be searched manually. Cross-sectional and longitudinal observational studies examining the association between informal caregiving for adults and loneliness or social isolation will be included. Studies focusing on grandchildren care or private care for chronically ill children will be excluded. Data extraction will include information related to study design, definition and measurement of informal caregiving, loneliness and social isolation, sample characteristics, statistical analysis and main results. The quality of the studies will be evaluated using the National Institutes of Health Quality Assessment Tool for Observational Cohort and Cross-Sectional Studies. Two reviewers will perform the selection of studies, data extraction and assessment of study quality. Figures and tables will be used to summarise and report results. A narrative summary of the findings will be provided. If data permit, a meta-analysis will be conducted.

Ethics and dissemination No primary data will be collected. Therefore, approval by an ethics committee is not required. We plan to publish our findings in a peerreviewed journal.

PROSPERO registration number CRD42020193099.

\section{INTRODUCTION}

Most individuals who need care prefer home care for as long as possible. ${ }^{12}$ A plausible explanation is that individuals who need care prefer to remain in familiar environments. ${ }^{34}$ In light of demographic ageing, the number of individuals in need of care is expected to rise. This emphasises the relevance of home care.

Informal caregiving (ie, provision of private care for relatives, friends or neighbours in need of care; often covering various tasks, eg, from assistance with household tasks to personal $\operatorname{care}^{5}$ ) is an important part of home

\section{Strengths and limitations of this study}

This will be the first systematic review focusing on the results regarding informal care, loneliness and social isolation.

- Quality assessment will be conducted for studies selected for inclusion in the review.

- Two reviewers: selection of the studies, data extraction and assessment of the study quality.

- Meta-analysis will be performed (if data permit).

Restricted to articles in German or English language

care. The vast majority of studies examining the consequences of informal caregiving have concentrated on health-related outcomes, such as depressive symptoms, and have mainly showed harmful effects for caregivers. ${ }^{6-10}$

Based on the caregiver stress model introduced by Pearlin et $a l^{11}$ caregiving can cover various stressors including caregiver burden. ${ }^{12-14}$ Depending on the coping resources, these stressors can also affect loneliness and social isolation. ${ }^{15}$ To date, some studies have explicitly examined social outcomes like loneliness and social isolation for caregivers, ${ }^{16-19}$ partially showing an association between informal caregiving and increased loneliness. However, it may also be the case that providing informal care can increase social network size and may therefore contribute to reduced loneliness or social isolation. Thus far, there is a lack of a review systematically synthesising evidence on the association between informal caregiving for adults, and loneliness as well as social isolation. Thus, a systematic review is required to establish the evidence base. Hence, the aim of this systematic review is to provide an overview of evidence from observational studies. This knowledge may assist in reducing loneliness and social isolation. This is important as these factors are associated with chronic conditions and longevity. ${ }^{20} 21$

Given the fact that informal caregiving must often be prioritised, the association 
between informal caregiving and increased loneliness and social isolation is plausible because informal caregiving may come at the expense of spending time with family and friends. ${ }^{22}$ Ultimately, this can result in increased self-reports of loneliness and social isolation. This is important since caregivers' experiences of loneliness can have adverse effects on emotional, social and physical well-being of them. ${ }^{23}$ However, it has been shown that the negative impact of caregiving can be mitigated (ie, through group-based leisure activities). ${ }^{22}$

It should be acknowledged that loneliness and social isolation are associated but are distinct concepts. ${ }^{24}$ Loneliness can be defined as the feeling that one's social network is of poorer quality or smaller than desired, ${ }^{25} 26$ whereas social isolation reflects a lack feeling that one belongs to the society. ${ }^{25-27}$ Moreover, (objective) social isolation can refer to a 'lack of contact with family, friends or other people ${ }^{, 28}$ (also see the Eligibility criteria section for further details). Both, loneliness and social isolation refer to social needs. ${ }^{29}$ Additional details regarding the terminology are, for example, provided by Holt-Lunstad ${ }^{30}$ or Dahlberg. ${ }^{31}$

\section{METHODS AND ANALYSIS}

The current review's methodology satisfied the Preferred Reporting Items for Systematic Reviews and MetaAnalyses (PRISMA) protocols guidelines. ${ }^{32}$ This review is registered with the International Prospective Register of Systematic Reviews. We intend to start our electronic search in May 2021 and plan to submit our systematic in a peer-reviewed journal in October 2021.

\section{Eligibility criteria}

We will perform a pretest (100 titles/abstract will be screened) prior to defining final eligibility criteria. Criteria will be refined as needed.

\section{Inclusion criteria}

Inclusion criteria for our systematic review are:

- Cross-sectional and longitudinal observational studies investigating the association between informal caregiving for adults ( $\geq 18$ years) and loneliness or social isolation.

- Assessment of key variables with established tools (eg, three item version of the UCLA Loneliness Scale V. $3^{33}$ or validated single item measures ${ }^{34}$ ).

- Studies in English or German language, published in a peer-reviewed, scientific journal.

It is worth noting that we will include studies dealing with perceived social isolation (eg, using the Bude and Lantermann scale ${ }^{35}$ ) and objective social isolation (eg, assessed by a small number or absence of contacts or relationships in different areas: individual, family, community and society ${ }^{313637}$ ) in our upcoming review.

It should be noted that perceived social isolation differs from loneliness. While these factors are correlated (eg, the De Jong Gierveld loneliness scale and the Bude and

\begin{tabular}{lc}
\hline Table 1 & Search strategy (Medline search algorithm) \\
\hline$\# 1$ & Informal careg* $^{*}$ \\
$\# 2$ & Family careg $^{*}$ \\
$\# 3$ & Private careg* $^{\star}$ \\
$\# 4$ & Spousal careg* \\
$\# 5$ & Parental careg* \\
$\# 6$ & $\# 1$ OR \#2 OR \#3 OR \#4 OR \#5 \\
$\# 7$ & Lonel $^{\star}$ \\
$\# 8$ & Social isolation \\
$\# 9$ & Social exclusion \\
$\# 10$ & \#7 OR \#8 OR \#9 ‘ \\
$\# 11$ & \#6 AND \#10 \\
\hline
\end{tabular}

Lantermann scale with about $\mathrm{r}=0.50$ in the German Ageing Survey), ${ }^{38}$ they do not measure the same construct. Moreover, they differ in their determinants and outcomes. ${ }^{39-41}$ For further details, see Hajek and König. ${ }^{38}$

\section{Exclusion criteria}

Exclusion criteria for our systematic review are:

- Studies focusing on grandchildren care.

- Studies focusing on private care for chronically ill children.

- Studies solely investigating samples with a specific disorder in the caregivers (eg, studies exclusively including caregivers with specific disorders such as depression).

We will search Medline, CINAHL and PsycInfo. Our search strategy for Medline is presented in table 1 (with no restrictions regarding the time and place of studies). Reference lists and citations of the studies included in our review will be searched manually by two reviewers (AH, BK).

\section{Data management}

Data will be imported into Endnote X7 (Clarivate Analytics, Philadelphia, Pennsylvania, USA), while (if possible), Stata V.16.0 (StataCorp) will be used for meta-analysis.

\section{Study selection process}

Two reviewers (AH, BK) will perform a title/abstract screening. Following this, the same two reviewers will screen full texts. Discussions will be held if opinions differ. A third party (H-HK) will be involved if consensus cannot be reached.

\section{Data collection process and data items}

Data extraction will be conducted by two reviewers $(\mathrm{AH}$, $\mathrm{BK})$. If required, we will involve a third party (H-HK). Data extraction will include study design, definition and measurement of main variables (ie, informal caregiving, loneliness and social isolation), sample characteristics, statistical approach and main findings. When important 
data cannot be extracted, or if clarification is required, we will contact the study authors.

\section{Assessment of study quality/risk of bias}

The study quality will be assessed using an appropriate quality assessment tool for observational cohort and cross-sectional studies (National Institutes of Health Quality Assessment Tool for Observational Cohort and Cross-Sectional Studies ${ }^{42}$ ). Two reviewers (AH, BK) will independently assess the quality of the studies. If needed, discussions will be held. A third party (H-HK) will be included, if agreement cannot be reached.

\section{Data synthesis}

A PRISMA flow diagram will be used to illustrate the process of study selection. The main results will be displayed via a narrative synthesis. Furthermore, we intend to perform a sensitivity analysis where we only analyse studies with a high quality. If possible, results will be categorised according to type of caregiving (eg, spousal or parental caregiving) or by outcome measure (eg, loneliness or social isolation). If the requirements for conducting a meta-analysis are fulfilled (eg, in terms of homogeneity in design and outcome measures), we will perform a meta-analysis. In further detail, extracted aggregated participant data will be quantitatively investigated by two individuals (AH, BK). In dependence of the fact whether there is significant heterogeneity, summary estimates (eg, standardised mean differences) will be estimated using a fixed-effect or random-effect meta-analysis (using the inverse variance method). The heterogeneity will be evaluated using the $\mathrm{I}^{2}$ test. A value of $\mathrm{I}^{2}>50 \%$ will be evaluated as indicative of high heterogeneity. ${ }^{43} \mathrm{In}$ such a case, a random-effects model will be used (fixed effect otherwise). Forest plots will also be used to visualise the degree of heterogeneity among studies.

Additional subgroup analyses by age of care-recipient and country of origin (eg, low-income and middle-income countries vs high-income countries) are intended.

\section{Patient and public involvement statement}

The present review protocol did not involve individual patients or public agencies.

\section{DISCUSSION}

To date, various studies have examined health consequences of informal caregiving while single studies $\left(\mathrm{eg}, \mathrm{see}^{44}\right)$ have revealed beneficial effects of informal caregiving, the majority of studies have shown harmful consequences $\left(\mathrm{eg}, \mathrm{see}^{6-10}\right)$. Some studies have also examined the association between informal caregiving and its consequences for loneliness and social isolation $\left(\mathrm{eg}, \mathrm{see}^{16-19}\right)$. Nevertheless, a systematic review, systematically synthesising these studies, is lacking. Consequently, the purpose of our systematic review is to give an overview of observational studies on the association between informal caregiving and loneliness or social isolation. Additionally, the quality of included studies will be assessed. Knowledge about an association between informal caregiving and loneliness or social isolation may be of particular importance to target individuals at risk for increased self-reports of loneliness or social isolation. Avoiding or responding to loneliness or social isolation may assist in maintaining health status of informal caregivers.

Our review may identify potential gaps in knowledge, such as a general lack of studies examining this association. Moreover, most of the existing studies may focus on loneliness, rather than social isolation. Depending on the tool used to quantify the outcome measures, the effects may vary. Furthermore, it is possible that evidence mainly stems from cross-sectional studies. In sum, this review may assist in guiding future research.

\section{Strengths and limitations}

This will be the first systematic review summarising empirical studies on the association between informal caregiving and loneliness, as well as social isolation. Several steps (ie, study selection, extracting the data and assessment of study quality) will be performed by two reviewers. Due to the possible heterogeneity of included studies, it is possible that a meta-analysis may not be feasible. We will restrict our search to articles published in peer-reviewed journals. While this may ensure a certain quality of the studies, other important studies may be excluded.

\section{ETHICS AND DISSEMINATION}

No primary data will be collected. Therefore, approval by an ethics committee is not required. We plan to publish our findings in a peer-reviewed journal.

Contributors The study concept was developed by AH, BK and H-HK. The manuscript of the protocol was drafted by $\mathrm{AH}$ and critically revised by $\mathrm{BK}$ and $\mathrm{H}-\mathrm{HK}$. The search strategy was developed by AH and H-HK. Study selection, data extraction and quality assessment will be performed by $\mathrm{AH}$ and $\mathrm{BK}$, with $\mathrm{H}-\mathrm{HK}$ as a third party in case of disagreements. All authors have approved the final version of the manuscript.

Funding The authors have not declared a specific grant for this research from any funding agency in the public, commercial or not-for-profit sectors.

Competing interests None declared.

Patient and public involvement Patients and/or the public were not involved in the design, or conduct, or reporting, or dissemination plans of this research.

Patient consent for publication Not required.

Provenance and peer review Not commissioned; externally peer reviewed.

Open access This is an open access article distributed in accordance with the Creative Commons Attribution Non Commercial (CC BY-NC 4.0) license, which permits others to distribute, remix, adapt, build upon this work non-commercially, and license their derivative works on different terms, provided the original work is properly cited, appropriate credit is given, any changes made indicated, and the use is non-commercial. See: http://creativecommons.org/licenses/by-nc/4.0/.

ORCID iD

André Hajek http://orcid.org/0000-0002-6886-2745 


\section{REFERENCES}

1 Hajek A, Lehnert T, Wegener A, et al. [Long-Term Care Preferences Among Individuals of Advanced Age in Germany: Results of a Population-Based Study]. Gesundheitswesen 2018;80:685-92.

2 Hajek A, Lehnert T, Wegener A, et al. Factors associated with preferences for long-term care settings in old age: evidence from a population-based survey in Germany. BMC Health Serv Res 2017;17:156

3 Prieto-Flores M-E, Forjaz MJ, Fernandez-Mayoralas G, et al. Factors associated with loneliness of noninstitutionalized and institutionalized older adults. J Aging Health 2011;23:177-94.

4 Victor CR. Loneliness in care homes: a neglected area of research? Aging health 2012;8:637-46.

5 Zwar L, König H-H, Hajek A. Do informal caregivers expect to die earlier? A longitudinal study with a population-based sample on subjective life expectancy of informal caregivers. Gerontology 2021:1-15.

6 Bauer JM, Sousa-Poza A. Impacts of informal caregiving on caregiver employment, health, and family. J Popul Ageing 2015;8:113-45.

7 Hajek A, König $\mathrm{H}-\mathrm{H}$. Informal caregiving and subjective well-being: evidence of a population-based longitudinal study of older adults in Germany. J Am Med Dir Assoc 2016;17:300-5.

8 Hajek A, König H-H. The Effect of Intra- and Intergenerational Caregiving on Subjective Well-Being--Evidence of a Population Based Longitudinal Study among Older Adults in Germany. PLoS One 2016;11:e0148916.

9 Hajek A, König $\mathrm{H}-\mathrm{H}$. The role of flexible goal adjustment in the effect of informal caregiving on depressive symptoms: evidence of a large population-based longitudinal study in Germany from 2002 to 2011. Qual Life Res 2017;26:419-27.

10 Kaschowitz J, Brandt M. Health effects of informal caregiving across Europe: a longitudinal approach. Soc Sci Med 2017;173:72-80.

11 Pearlin LI, Mullan JT, Semple SJ, et al. Caregiving and the stress process: an overview of concepts and their measures. Gerontologist 1990;30:583-94.

12 Braich PS, Lal V, Hollands S, et al. Burden and depression in the caregivers of blind patients in India. Ophthalmology 2012;119:221-6.

13 Gallicchio L, Siddiqi N, Langenberg P, et al. Gender differences in burden and depression among informal caregivers of demented elders in the community. Int J Geriatr Psychiatry 2002;17:154-63.

14 Hanly P, Maguire R, Balfe M, et al. Burden and happiness in head and neck cancer carers: the role of supportive care needs. Support Care Cancer 2016;24:4283-91.

15 Zwar L, König H-H, Hajek A. Psychosocial consequences of transitioning into informal caregiving in male and female caregivers: findings from a population-based panel study. Soc Sci Med 2020;264:113281.

16 Brodaty H, Hadzi-Pavlovic D. Psychosocial effects on carers of living with persons with dementia. Aust N Z J Psychiatry 1990;24:351-61.

17 Curvers N, Pavlova M, Hajema K, et al. Social participation among older adults $(55+)$ : results of a survey in the region of South Limburg in the Netherlands. Health Soc Care Community 2018;26:e85-93.

18 Hajek A, König H-H. Impact of informal caregiving on loneliness and satisfaction with leisure-time activities. findings of a populationbased longitudinal study in Germany. Aging Ment Health 2019;23:1539-45.

19 Stoltz P, Udén G, Willman A. Support for family carers who care for an elderly person at home - a systematic literature review. Scand J Caring Sci 2004;18:111-9.

20 Rico-Uribe LA, Caballero FF, Martín-María N, et al. Association of loneliness with all-cause mortality: a meta-analysis. PLoS One 2018;13:e0190033.

21 Valtorta NK, Kanaan M, Gilbody S, et al. Loneliness and social isolation as risk factors for coronary heart disease and stroke: systematic review and meta-analysis of longitudinal observational studies. Heart 2016;102:1009-16.

22 Schüz B, Czerniawski A, Davie N, et al. Leisure time activities and mental health in informal dementia caregivers. Appl Psychol Health Well Being 2015;7:230-48.

23 Gray TF, Azizoddin DR, Nersesian PV. Loneliness among cancer caregivers: a narrative review. Palliat Support Care 2020;18:359-67.

24 Victor C, Scambler S, Bond J, et al. Being alone in later life: loneliness, social isolation and living alone. Rev Clin Gerontol 2000;10:407-17.

25 Petersen N, König H-H, Hajek A. The link between falls, social isolation and loneliness: a systematic review. Arch Gerontol Geriatr 2020;88:104020.

26 Wenger GC, Davies R, Shahtahmasebi S, et al. Social isolation and loneliness in old age: review and model refinement. Ageing Soc 1996;16:333-58.

27 Nicholson NR. Social isolation in older adults: an evolutionary concept analysis. J Adv Nurs 2009;65:1342-52.

28 Marczak J, Wittenberg R, Doetter L. Preventing social isolation and loneliness among older people. Eurohealth 2019;25:3-5.

29 Bunt S, Steverink N, Olthof J, et al. Social frailty in older adults: a scoping review. Eur J Ageing 2017;14:323-34.

30 Holt-Lunstad J. Social isolation and health. In: Health affairs brief, 2020.

31 Dahlberg L, McKee KJ, Frank A, et al. A systematic review of longitudinal risk factors for loneliness in older adults. Aging Ment Health 2021:1-25.

32 Shamseer L, Moher D, Clarke M. Preferred reporting items for systematic review and meta-analysis protocols (PRISMA-P) 2015: elaboration and explanation. BMJ 2015;349:g7647.

33 Hughes ME, Waite LJ, Hawkley LC, et al. A short scale for measuring loneliness in large surveys: results from two population-based studies. Res Aging 2004;26:655-72.

34 Nersesian PV, Han H-R, Yenokyan G, et al. Loneliness in middle age and biomarkers of systemic inflammation: findings from midlife in the United States. Soc Sci Med 2018;209:174-81.

35 Bude H, Lantermann E-D. Soziale exklusion und exklusionsempfinden. KZfSS Kölner Zeitschrift für Soziologie und Sozialpsychologie 2006;58:233-52.

36 Cornwell EY, Waite LJ. Measuring social isolation among older adults using multiple indicators from the NSHAP study. J Gerontol B Psychol Sci Soc Sci 2009;64 Suppl 1:i38-46.

37 Dahlberg L. Loneliness during the COVID-19 pandemic. Aging Ment Health 2021:1-4.

38 Hajek A, König H-H. Do loneliness and perceived social isolation reduce expected longevity and increase the frequency of dealing with death and dying? longitudinal findings based on a nationally representative sample. J Am Med Dir Assoc.

39 Menec VH, Newall NE, Mackenzie CS, et al. Examining individual and geographic factors associated with social isolation and loneliness using Canadian longitudinal study on aging (CLSA) data. PLoS One 2019;14:e0211143.

40 Schrempft S, Jackowska M, Hamer M, et al. Associations between social isolation, loneliness, and objective physical activity in older men and women. BMC Public Health 2019;19:74.

41 Steptoe A, Shankar A, Demakakos P, et al. Social isolation, loneliness, and all-cause mortality in older men and women. Proc Natl Acad Sci U S A 2013;110:5797-801.

42 Health. Nlo. National Heart Lung, and Blood Institute. Study quality assessment tools, 2018

43 Lipsey M, Wilson D. Practical meta-analysis. Thousand Oaks: SAGE Publications, 2000

44 Zwar L, König $\mathrm{H}-\mathrm{H}$, Hajek A. The impact of different types of informal caregiving on cognitive functioning of older caregivers: evidence from a longitudinal, population-based study in Germany. Soc Sci Med 2018;214:12-19. 